\title{
Kernos
}

Revue internationale et pluridisciplinaire de religion grecque antique

$16 \mid 2003$

Varia

\section{Les traits de Zeus Pandoros, selon l'Hymne à Zeus de Cléanthe}

Maria Protopapas-Marnelli

\section{(2) OpenEdition}

\section{Journals}

Édition électronique

URL : http://journals.openedition.org/kernos/822

DOI : $10.4000 /$ kernos.822

ISSN : 2034-7871

Éditeur

Centre international d'étude de la religion grecque antique

Édition imprimée

Date de publication : 1 janvier 2003

Pagination : 191-195

ISSN : 0776-3824

Référence électronique

Maria Protopapas-Marnelli, « Les traits de Zeus Pandoros, selon l'Hymne à Zeus de Cléanthe », Kernos [En ligne], 16 | 2003, mis en ligne le 14 avril 2011, consulté le 19 avril 2019. URL : http:// journals.openedition.org/kernos/822 ; DOI : 10.4000/kernos.822 


\section{Les traits de Zeus Pandoros, selon l'Hymne à Zeus de Cléanthe}

Je me propose de dégager les traits constitutifs de la théologie stoïcienne, tels qu'ils se présentent dans l'Hymne à Zeus de Cléanthe. Pour ce faire, j'ai divisé mon propos en trois parties qui portent respectivement sur : (1) le rôle de la théologie dans le système stö̈cien; (2) la fonction de l'Hymne à Zeus à l'intérieur de la théologie du Portique; (3) le rapport de la divinité à la vie du sage stoïcien moyennant l'invocation de Zeus comme Pandoros.

\section{Le rôle de la théologie}

Invoquer dieu constitue le point culminant de toute théologie à l'époque hellénistique. L'homme se laisse entraîner vers une paideia qui, dans le domaine de l'astronomie', lui révèle l'explication des phénomènes célestes ${ }^{2}$ et, dans le domaine de la théologie, lui prouve la puissance d'un dieu unique qui, sous des traits aussi bien individuels que cosmiques, sert d'appui à l'homme grec ou hellénisant, qui tente de se réorienter dans l'étendue des nouveaux domaines des empires post-alexandrins. « Astronomie et monothéisme sont les bagages intellectuels de quiconque aspire à être cultivé $»^{3}$. En ce qui concerne les Stö̈ciens, l'accent est mis sur la division tripartite de la philosophie en physique, en morale et en logique, conçues comme un ensemble inséparable, à tel point que, si l'on essayait de rompre cette unité, tout le système se désagrégerait ${ }^{4}$. Néanmoins, chacun des domaines de cet ensemble est, à son tour, divisé en sciences autonomes, et pourtant constitutives du corps qu'est lá philosophie stö̈cienne.

Parmi les premiers scholarques de l'École, seul Cléanthe a osé insérer et intégrer la théologie comme science autonome dans la division de la philosophie stö̈cienne ${ }^{5}$ et démontrer, par le dynamisme de ses vers, le rôle ultime de la théologie scientifique qui s'accorderait, sans se confondre pour autant, à la théologie poétique de la religion populaire $^{6}$. Par la suite, Chrysippe, selon le témoignage de Plutarque ${ }^{7}$, considéra la théologie comme constituant les $\tau \varepsilon \lambda \varepsilon \tau \alpha i$ de la physique, c'est-à-dire la doctrine dominée par la physique, mais qui, en même temps, conduit à une initiation aux ultimes

1 Cf. Cicéron, De divin. II, 42, 88; cf. aussi D. BABU', La religion des philosopbes grecs, Paris, 1974, p. 190 et n. 4.

Cf. E. MaAs, Commentariorum in Aralum reliquiae, Berlin/Neüköln, 1958.

A.-J. Festugière, Études de religion grecque et bellénistique, Paris, 1972, p. 121.

4 Diogìne Lä̈rce (désormais D.L.) VII, 47.

D.L. VII, 39-40.

F. OGÉreau, Essai sur le système philosophique des Stö̈ciens, Paris, 1885, p. 243.

7 Plutarque, De st. repugn., 1035a-b. 
mystères de la réalité ${ }^{8}$. Cette réalité cependant ne se manifeste pas uniquement à travers la religion traditionnelle des Grecs ou celle des autres peuples qui, ultérieurement, ont pu facilement incorporer le culte de leurs propres divinités dans celui des divinités populaires helléniques". Les voyages entrepris par les Grecs, à des fins commerciales, contribuèrent à développer encore leur largeur d'esprit et ils furent impressionnés par de nouveaux us et coutumes, au point de vouloir les mêler aux rites de leur propre religion.

Ce mélange se transforma en passant du cadre de l'éthique à la physique

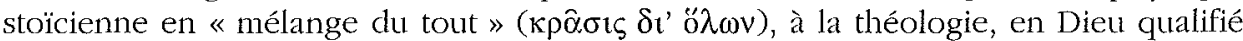
de plusieurs noms ( $\pi \circ \lambda v \omega ́ v v \mu \circ$ ) regroupant les qualités de toutes les divinités traditionnelles. C'est dans ce contexte social prolongé dans une physique qu'il faut concevoir la théologie des Stoïciens. Si les divers peuples sont appelés à se fondre en un même peuple et à constituer une même cité, c'est qu'en vérité une telle cité existe déjà : c'est la Cité du Monde, régie par le Dieu cosmique. Les dieux particuliers ne sont que des manifestations locales de la même et unique divinité répandue dans

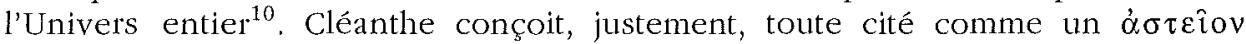
oikntńpıov, une «maison des hommes de bien » où les citoyens vivent en harmonie,

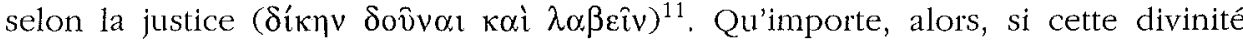
s'appelle Zeus, Nature ou Logos. Les Stö̈ciens ont su situer leur dieu dans un milieu profondément naturaliste avant de puiser dans les mythes qui accompagnaient les divinités populaires. Le mythe peut toujours refléter un enseignement sous des aspects divers ${ }^{12}$. Et, dans la mesure où il est formé chez l'homme à son insu, la création mythique de la vie ou les qualités d'un dieu portent l'empreinte de tout un peuple ${ }^{13}$. Aussi cet arsenal ancestral a-t-il pu servir aux Stoïciens d'appui à leur conception selon laquelle les mythes et les noms des divinités étaient capables de révéler des sens cachés ${ }^{14}$. Ne pas s'éloigner de la tradition ne signifie aucunement, pour les philosophes du Portique, ne pas inventer, ne pas chercher et, finalement, ne pas créer une théorie nouvelle relative au divin. «Dieu, affirment-ils, est un vivant immortel, raisonnable, parfait, intelligent, bienheureux, incapable d'admettre en lui aucun mal, ordonnant par sa providence le monde et les choses qui sont dans le monde; mais il n'a pas forme humaine; il est le démiurge de l'Univers en tant que père de toutes choses, à la fois en général et par la partie de lui-même qui pénètre toutes choses, partie qui est appelée de plusieurs noms, selon les pouvoirs qu'il exerce $»^{15}$.

8 Cf. M. FreDe, La théologie stolicienne, conférence inédite, prononcée dans le cadre des travaux du Séminaire du Centre Léon Robin, Paris IV, Sorbonne, mai, 2000.

9 Cf. P.-M. SChuHL, Les Stö̈ciens, Paris, 1962, p. LVI : « c'est grâce à ce détachement total de toute patrie, de toute tradition locale, que le stoïcisme a dû, en partie, sa diffusion "; $c f$. ibid, note (a).

10 Festugière, o.c. (n. 3), p. 127

11 SVF I, 587 (= STOв., Ecl. II, 7, 111, p. 103, 12 W.).

12 D. Babut, Plutarque et le stolicisme, Paris, 1969 , p. 370.

13 Cf. C. LÉvi-Strauss, Le mythe et le sens (tr. gr. par V. Athanassopoulos), Athènes, Kardamitsa, 1989 , p. 23.

14 Cf. R. Hoven, Stoïcisme et Stö̈ciens face au problème de l'au-delà, Paris, 1971, p. 77.

15 D.L. VII, 147. 


\section{L'Hymne à Zeus et son rapport avec la théologie du Portique}

Selon les Stoïciens, Dieu est, on l'a vu, dépourvu de forme humaine ${ }^{16}$ et l'on connaît l'attitude de Zénon face aux constructeurs des temples ${ }^{17}$. Cependant, pour que le polythéisme populaire demeure vivant, nos philosophes s'appuyent sur les divinités traditionnelles tout en leur attribuant une fonction et un visage différents. Cette méthode d'approche des dieux populaires, qui consiste en l'interprétation allégorique de leurs noms ainsi que des mythes qui en accompagnent l'existence, était déjà connue dans l'Antiquité ${ }^{18}$. Mais avec les Stöiciens, qui veulent défendre l'éducation de leur époque contre les outrances des Cyniques, l'interprétation allégorique devient une manière de concevoir la divinité. Ils admettent alors, dans leur théologie, la poésie ancienne, plus ou moins «corrigée » et interprétée allégoriquement ${ }^{19}$. Cléanthe affirme que, moyennant l'allégorie, les passions humaines se calment. L'explication des notions qu'ils introduisent en philosophie et l'enseignement de leur doctrine deviennent plus intelligibles ${ }^{20}$. Selon eux, les idées sont plus facilement véhiculées par l'intermédiaire du discours poétique, étant donné qu'elles demeurent cachées dans les légendes, les mythes et la fantaisie pure. Cette méthode fut introduite par Zénon et Cléanthe, avant d'être perfectionnée, ultérieurement, par Chrysippe $^{21}$. Grâce à l'interprétation, les trois scholarques mirent à profit Homère et les autres poètes pour mieux souligner le rôle de la logique comme se référant au principe ultime du Monde. Aussi, dans l'Hymne à Zeus, l'adjectif « polyonyme» annonce-t-il que Dieu comprend les qualités de toutes les divinités traditionnelles ${ }^{22}$.

Dans l'hymne, Zeus est évoqué en hexamètres par des noms que les Stoïciens lui attribuaient, et par des symboles à connotation stoïcienne. Cléanthe nous prodigue une leçon de théologie scientifique. Selon lui, Zeus tout-puissant est glorifié comme dominateur de la Nature et créateur cosmique. Il est censé être accompagné de la Loi universelle. Par la puissance de sa foudre, il dirige la raison commune. Seul le mal moral, dû à l'ignorance, échappe à sa providence. En s'éloignant de la raison divine $(\log o s)$, l'ignorant se dirige vers l'acquisition de biens dépourvus de valeur morale, et, loin d'atteindre le bonheur, se rend malheureux. Dieu seul a la puissance de transformer le mal en bien, d'éclairer les ténèbres de l'ignorance humaine et de ramener sur le droit chemin celui qui s'en égare, afin qu'il partage avec lui la Raison divine. En effet, les mortels ont le droit d'invoquer l'intervention divine, en même temps qu'ils glorifient la puissance et les ouvres de Dieu, tant ils sont liés à lui par des liens de parenté; et doués, grâce à lui, du logos, c'est à juste titre qu'ils l'appellent «père ». Cléanthe termine sa leçon de théologie par la prière finale dans laquelle il fait

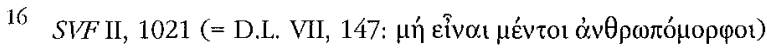

17 SVF I, 264 (= Clém. Alex , Strom. V, 12, 76, p. 691 P.) et SVF I, 265 (= Orig., C.Cel. I, 5, Vol. I, p. 59,3 Kö., p. 324 Del.); cf aussi BabuT, o.c. (n. 1), p. 176.

18 Cf. p. ex. dans le Cratyle.

19 M. Dragona-Monachou, "The Hymn to Zeus and the Golden Verses, Cleanthes' Poetical Theology and the Orphico-Pythagorean tradition" [en grec avec rés, en anglais], $\Phi_{\imath} \lambda \sigma^{\sigma} \sigma \varphi{ }^{\prime} \alpha 1$ (1971), p. 339-378, spéc. p. 352.

20 SVF I, 526 (= Apol.., Soph. Lex. Homer, 114 Bekker s.v.).

21 BABUT, o.c. (n. 12), p. 369.

22 Cf. R. PFeIfFer, History of Classical Scbolarship, from the Beginnings to the End of the Hellenistic Age (tr. gr. par P. Xénos), Athènes, 1972, p. 283; cf. aussi Babut, o.c. (n. 1), p. 186. 
état, en plus de la parenté, de l'assimilation des hommes au divin ${ }^{23}$, se manifestant à travers le respect dont font preuve les uns et les autres pour la loi universelle qui gouverne le monde $e^{24}$.

\section{Zeus pandoros, dans la vie du sage stoïcien}

Cette appellation n'est présentée en aucun autre hymne à Zeus dont les qualités sont déjà évoquées aux vers 1-16. Le problème que Cléanthe pose par la suite (v. 1731) porte sur les crimes de folie que les méchants commettent par ignorance. Zeus est toutefois capable de réduire les excès et de mettre en ordre ce qui est en désordre en vue du bon fonctionnement de l'Univers. Il a fondu les biens et les maux en un tout harmonieux. Grâce à son intervention, tout a été intégré à nouveau dans la chaîne des événements cosmiques, momentanément interrompue à cause des crimes des ignorants. Cléanthe procède à la description psychique de ces derniers qui, ne sachant faire un bon usage de la raison, du don que Dieu leur a accordé, sont malheureux. Ils désirent des biens qui, pour un stoïcien, sont des maux ${ }^{25}$, tels la gloire, les voluptés du corps, l'acquisition de biens par la fraude ${ }^{26}$. Quand ils n'arrivent pas à les acquérir, leur âme, mue par le mouvement déraisonnable qu'est le vice, se rend malheureuse ${ }^{27}$. Le philosophe d'Assos, qui enseigne que la seule nature qu'il faut suivre est la nature universelle et non point notre nature particulière ${ }^{28}$, souligne que c'est uniquement par manque de sagesse que les méchants agissent de la sorte. S'ils

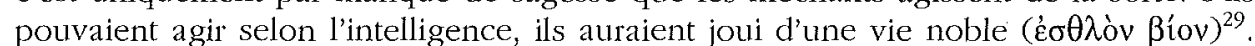
Cléanthe affirme que, de mauvais, on peut devenir vertueux ${ }^{30}$ et que, pour atteindre le bonheur dans sa vie, l'homme doit agir moralement, ce qui le conduit à la vertu.

Nous sommes, dès lors, en mesure de comprendre la raison pour laquelle notre

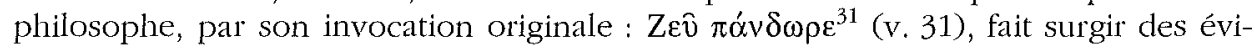
dences oubliées et conduit son auditoire au monde du mythe et de la tradition, dont

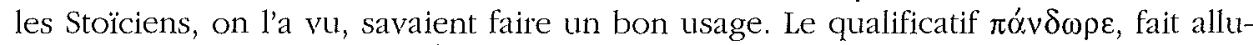
sion à Pandora, l'épouse d'Épiméthée, créée par la volonté de Zeus exclusivement pour l'homme, et dotée de tous les dons divins ${ }^{32}$ mais également porteuse de tous les

23 Cf. SVF II, 1076 (= PHILod., De piet., c. 11 (DDG 545 b 12); cf. D.L. VII, 119 : « Les sages sont divins, parce qu'ils ont Dieu en eux-mêmes. »

24 Cf. R.P. Festugière, La révélation d'ftermès Trismégiste, II. Le dieu cosmique, Paris, 1949, p. 330 .

25 D.L. VII, 97.

26 V. 25-29, tr. Festugière.

27 D.L. VII, 110.

28 D.L. VII, 89

29 V. 25, tr. Festugière.

30 D.L. VII, 91 : « la vertu peut être enseignée. »

31 Cf. Chr. Brown, "Cleanthes' Hymn to Zets 32", LCM 1 (Jan. 1990), p. 2-5: « L'adjectif

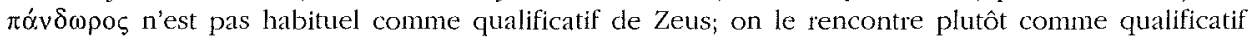
d'une divinité féminine. Ici, cet adjectif renvoie plutôt à la théorie stoïcienne qui iclentifie Zeus au destin » (tr. de l'alteur).

32 Hésıode, Travalux, 59-82. 
malheurs pour le genre humain. Hésiode, on le sait, fait de Pandora ${ }^{33}$ cette femme, conçue comme un mal absolu ${ }^{34}$, pourtant indispensable pour la reproduction biologique des humains, qui introduisit en même temps l'art du tissage, un art qu'Athéna lui avait appris pour le lui faire enseigner aux hommes ${ }^{35}$. Or, nécessité et malheur, bien et mal ${ }^{36}$ coexistent chez le même personnage mythique. On constate une réconciliation de ces forces adverses dans les vers où Hésiode décrit les plans de vengeance de Zeus : « en place du feu je leur ferai présent d'un mal en qui tous, du fond du coeur, se complairont à entourer d'amour, leur propre malheur » ${ }^{37}$. Dans les vers de Cléanthe, l'homme est toujours représenté comme étant hanté par la recherche du bien et de l'utile : c'est parce que l'homme est toujours en quête de l'utile, qu'il risque de sombrer dans l'erreur et ce, en raison d'une mauvaise évaluation des situations, due à son incapacité de bien raisonner.

Pour conclure, j'aurai recours, une fois de plus, au personnage de Pandora (donatrice de toutes choses), cette créature au coeur artificieux, faite de terre et d'eau, "piège profond et sans issue », selon le poète de la Théogonie (v. 589), mais dont l'esprit ne peut être considéré comme totalement négatif, surtout à l'intérieur de la mythologie grecque, qui fait des dieux et des héros importants les promoteurs de quelque ruse identifiée à la sagesse ${ }^{38}$. En l'occurrence, l'art du tissage aurait été inventé par Athéna pour le bien de l'homme, diffusé par Pandora, également porteuse de malheurs. Dans la pensée de Cléanthe, cela représente la solution au problème du mal qui coexiste avec le bien. L'interprétation de cette idée trouve sa justification dans le mythe de Pandora, de cette présence féminine si ambiguë. Cléanthe prie Zeus

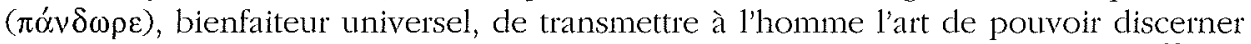
ce qui est vraiment utile dans sa vie, de lui manifester, comme dans le mythe ${ }^{39}$, les moyens de jouir de ses dons, et, tout comme il aurait appris, par le truchement de Pandora à ses ancêtres l'art du tissage, d'apprendre aux hommes à tisser le voile de leur vie selon la sagesse de la raison clivine.

Centre de Philosophie ancienne

Maria PRotoPAPAS-MARNELLI

Anagnostopoulou, 14

GR - 10673 ATHÈNES

33 Pour une analyse du mot pandoros dans l'hymne, cf. Festugière, La révélation... o.c. (n. 24), p. 321; $c f$. aussi A.W. JAmes, "The Zeus Hymns of Cleanthes and Aratus", Allichthon 6 (1972), p. 2837, spéc. p. 33.

34 P. Lévêque, "Pandora ou la terrifiante féminité", Kernos 1 (1988), p. 49-62, spéc. p. 54; cf. aussi H.J. Rose, A Handbook of Greek Mytbology, Methuen \& Co., 1974, p. 55.

35 J.M. Hurwit, "Beautiful Evil: Pandora and the Athena Parthenos", A/A 99 (1995), p. 171-186, spéc. p. 183.

36 Cf. ant. Pandora, LIMC VI, 1 (1994), p. 163-166.

37 Travaux, 57 sq. (trad. P. Lévêque).

38 LÉVÊQUE, l.c. (n. 34), p. 56.

39 Nous ne partageons pas ici l'avis de Ph. DE LACY, "Stoic views of poetry" (trad. grecque par A. Zamanis), Eкnßódos 1 (1978), p. 47-76, spéc. p. 66, selon lequel l'élément du mythe dans l'hymne est réduit au minimum. 